NEW ZEALAND JOURNAL OF MATHEMATICS

Volume 52 (2021), 727-731

https://doi.org/10.53733/189

\title{
EMBEDDING HEEGAARD DECOMPOSITIONS
}

\author{
Ian Agol and Michael Freedman
}

(Received 22 November, 2021)

\begin{abstract}
A smooth embedding of a closed 3-manifold $M$ in $\mathbb{R}^{4}$ may generically be composed with projection to the fourth coordinate to determine a Morse function on $M$ and hence a Heegaard splitting $M=X \cup_{\Sigma} Y$. However, starting with a Heegaard splitting, we find an obstruction coming from the geometry of the curve complex $C(\Sigma)$ to realizing a corresponding embedding $M \hookrightarrow \mathbb{R}^{4}$.
\end{abstract}

We will miss Vaughan Jones, not only for his great mathematics, but for his joi de vivre. We recall fondly a conference in Taipa, New Zealand organized by Vaughan and the NZMRI which had ample mathematics accompanied by barbecued fresh mussels, hiking, and windsurfing. His legacy will be carried on by those with a love for both mathematics and the outdoors.

\section{Introduction}

The tools for showing that a closed 3-manifold $M$ does not smoothly embed in $\mathbb{R}^{4}$ seem rather primitive. There does not seem to be any $M$ which embeds in some integral homology 4 -sphere $\Sigma^{4}$ and is known not to embed in $\mathbb{R}^{4}$. But tools for this would be highly desirable since Budney and Burton's [1, §4] 3-manifold survey turns up four examples of closed 3-manifolds $M$ embedding in a homotopy 4-sphere for which no embedding in $\mathbb{R}^{4}$ is known. This raises the possibility that 3 -manifold embeddings could be used to detect exotic structures.

Our goal here is to find a bridge between the rich subject of surface dynamics, e.g. the mapping class group, and embeddability in the hope that the coordinate structure of $\mathbb{R}^{4}$ will make an essential appearance. We are partially successful. We find a robust connection between the very coarse "handlebody metric" $d_{H}$ on the curve complex $C(\Sigma)$ recently studied in $[\mathbf{6}]$ and embeddability of the corresponding Heegaard decompositions. Said another way, we turn $d_{H}$ into an obstruction to embedding $f: M \hookrightarrow \mathbb{R}^{4}$ with the fourth coordinate already prescribed. We have known this result since 2012 but have been unable to accomplish the obvious next step: find some residual obstruction which is independent of the fixed $4^{\text {th }}$ coordinate function, i.e. define a true embedding obstruction based on surface dynamics. Since [6] has now appeared in print and our argument provides a simple application, possibly with yet unrealized potential, we present it here.

Assume that we have been given a Morse function $f: M \rightarrow \mathbb{R}$, how can we show it is not the fourth coordinate of any embedding in $\mathbb{R}^{4}$ ? Actually, the obstruction we formulate will not make use of the entire data of a Morse function but merely the Heegaard decomposition $M=X \cup_{\Sigma} Y$ canonically determined (up to isotopy) by $f$. The handlebody $X$ is a neighborhood of the ascending manifolds of critical points of index $=2$ and 3 and $Y$ is a neighborhood of the descending manifold of critical points of index 0 and 1 . 
Our chief tool is the curve complex $C(\Sigma)[4]$ and its metrics. The vertices of $C(\Sigma)$ are isotopy classes of simple closed curves (sccs) on $\Sigma$, and Hempel [5] introduced the metric $d$, the largest metric where disjoint sccs have distance $=1$. We will exploit a much coarser metric $d_{H}$, "handlebody distance," defined as the largest metric where any two sccs bounding disks in the same handlebody $H, \partial H=\Sigma$, have distance $=1$. This distance is easily seen to be quasi-isometric to the "electrification metric" $d_{E}$ recently introduced in $[6]$, where it is proved that $\operatorname{diam}_{d_{E}}(C(\Sigma))=\infty$, for genus $\Sigma \geq 2$. So for us, a key fact will be

$$
\text { for genus } \Sigma \geq 2, \operatorname{diam}_{d_{H}}(C(\Sigma))=\infty \text {. }
$$

Let $D(X) \subset C(\Sigma)$ be the set of sccs bounding disks in the handlebody $X=\partial \Sigma$. We prove the following:

Theorem 3.1. If $M$ embeds in smoothly $\mathbb{R}^{4}$ with one (say, the fourth) coordinate determining the Heegaard decomposition $M=X \cup_{\Sigma} Y$, then $d_{H}(D(X), D(Y)) \leq 2$.

We actually supply two proofs (using slightly different techniques, yielding slightly different constants, and supporting different generalizations).

\section{Ambient Morse Theory}

This section recalls an "ambient" version of Morse theory appropriate to embedded submanifolds $M^{3} \hookrightarrow \mathbb{R}^{4}$.

When speaking of an embedding $f: M \hookrightarrow \mathbb{R}^{4}$, we will feel free to change the target space to $S^{4}$ or $S^{3} \times \mathbb{R}$, by adding or deleting a point, without renaming the map or calling other attention to the change.

Suppose we are given a codimension-1 smooth embedding $g: M^{3} \hookrightarrow \mathbb{R}^{4}$ of a closed connected 3 -manifold with fourth coordinate $g_{4}=f$. Using only elementary general position argument, one constructs an isotopy from $g$ to $g^{\prime}$ with the critical points of the fourth coordinate $g_{4}^{\prime}$ occurring in order (higher index critical points take larger values). Such Morse functions will be called ordered.

Some choices are made in this procedure which could influence the order of handle attachments but not the diffeomorphism type of the Heegaard decomposition $M=X \cup_{\Sigma} Y$, where $Y=\cup$ (handles of index $=0,1$ ) and $X=\cup$ (handles of index $=2,3)$. The topology of $X(Y)$ relative to $\Sigma$ is, however, independent of any choices.

Lemma 2.1. If $M$ is an embedding in $\mathbb{R}^{4}$ and $M=X \cup_{\Sigma} Y$ is the Heegaard splitting associated to the fourth coordinate with $\Sigma \subset \mathbb{R}^{3} \times 0 \subset \mathbb{R}^{4}$, then provided genus $(\Sigma) \geq 1, D(X)$ and $D(Y)$ both contain at least one scc which compresses in $\mathbb{R}^{3} \times 0$.

Lemma 2.2. If $N$ is a 3-manifold (compact or non-compact) and there is an embedding $M \hookrightarrow N \times \mathbb{R}$ inducing a Heegaard splitting $M=X \cup_{\Sigma} Y, \Sigma \subset N \times 0$, then provided genus $(\Sigma) \geq 1, D(X)$ and $D(Y)$ both contain at least one scc which compresses in $N \times 0$.

Proof. Both lemmas are proven by sliding $\Sigma$ up and down the gradient lines of the Morse function until the first collapse of an essential scc in $\Sigma$ is observed. 


\section{Two Distance Estimates}

Proof of Theorem 3.1. We may compactify horizontal slices to consider the embedding of $M$ as into $S^{3} \times \mathbb{R} . M=X \cup_{\Sigma} Y$ and we may assume

$$
\Sigma \subset S^{3}=S^{3} \times 0 \subset S^{3} \times \mathbb{R}
$$

and, since lens spaces do not embed in $\mathbb{R}^{4}$, without loss of generality that $g(\Sigma) \geq 2$. Note that $\Sigma \subset S^{3}$ may not be a Heegaard surface for $S^{3}$ but by Lemma 2.1 must contain at least one scc of $D(X)$ and one scc of $D(Y)$ (which might be identical) which compress into $S^{3}$.

Notation. $S^{3}=A \cup_{\Sigma} B$ and $V \subset A$ and $W \subset B$ are maximal compression bodies for $\Sigma$ in $A$ and $B$, respectively (see [2] for the definition of a compression body).

At most one of $V$ and $W$ is a product collar (since $S^{3}$ is non-Haken). By Fox [3, Main Theorem (1)], $V \cup_{\Sigma} W$ may be re-embedded into $S^{3}$ so that the complementary regions $P$ and $Q$ lying in $A$ and $B$ respectively are unions of handlebodies and consequently $H=P \cup_{\partial_{-} V} V$ and $J=Q \cup_{\partial_{-} W} W$ are also handlebodies and $S^{3}=H \cup_{\Sigma} J$ is a Heegaard decomposition. By Waldhausen [7], this decomposition is standard; thus, $D(H) \cap D(J) \neq \emptyset$, so

$$
\operatorname{diam}_{d_{H}}(D(H) \cup D(J))=2 .
$$

(From the definition of $d_{H}$, $\operatorname{diam}_{d_{H}}(D(H) \cup D(J)) \leq 2$, but since $D(H) \cup D(J)$ contains a pair of sccs with homological intersection number $=1, \operatorname{diam}_{d_{H}}(D(H) \cup$ $D(J))>1$.)

But as noted above, both $D(X)$ and $D(Y)$ must meet $D(H) \cup D(J)$, so

$$
d_{H}(D(X), D(Y)) \leq 2 .
$$

Theorem 3.2. Let $N$ be a closed, reducible, 3-manifold containing no incompressible surface, or $S^{3}$. Let $M$ be a closed 3-manifold embedded in $N \times \mathbb{R}$, with the $\mathbb{R}$ coordinate inducing a Heegaard splitting $M=X \cup_{\Sigma} Y$. We have $d_{H}(D(X), D(Y)) \leq$ 3.

Proof. Use ambient Morse theory to produce $\Sigma \subset N \cong N \times 0$ with $X \subset N \times[0, \infty)$ and $Y \subset N \times(-\infty, 0]$. By Lemma 2.2, there is at least one scc of $D(X)$ and $D(Y)$ compressing in $N$. (It might be the same curve.) Let $\Sigma$ separate $N$ as $N=A \cup_{\Sigma} B$ and let $V \subset A$ and $W \subset B$ be the maximal compression bodies on the two sides of $\Sigma$. Since $N$ contains no incompressible surface, at most one of $V$ and $W$ is a product collar.

Suppose neither $V$ nor $W$ is a collar. Then it must follow that the $d$-distance between the sets of compression disks, $d(D(V), D(W)) \leq 1$. For otherwise the generalized Heegaard decomposition $V \cup_{\Sigma} W$ would be strongly irreducible, so the main theorem of $\mathrm{C}-\mathrm{G}[\mathbf{2}]$ implies that $V \cup_{\Sigma} W$ is irreducible and has either incompressible or empty boundary. This boundary must be non-empty by the reducibility of $N$. But since $\partial_{-} V \hookrightarrow(A \backslash V)$ and $\partial_{-} W \hookrightarrow(B \backslash W)$ are incompressible (by maximality of $V$ and $W$ ), we would conclude $\partial\left(V \cup_{\Sigma} W\right) \hookrightarrow N$ is incompressible, 
contradicting the assumption of no incompressible surface. However, by Lemma 2.2, we have

$$
D(X) \cap(D(V) \cup D(W)) \neq \emptyset \neq D(Y) \cap(D(V) \cup D(W))
$$

Since $d_{H}(D(V), D(W)) \leq d(D(V), D(W)) \leq 1$ and $\operatorname{diam}_{d_{H}}(D(V)), \operatorname{diam}_{d_{H}}(D(W))$ $\leq 1$, we conclude by the triangle inequality that $d_{H}(D(X), D(Y)) \leq 3$.

Now suppose one of $V$ or $W$, say $V$, is a collar. Then

$$
D(X) \cap D(W) \neq \emptyset \neq D(Y) \cap D(W),
$$

so $d_{H}(D(X), D(Y)) \leq 1$.

Maher and Schleimer studied [6] a metric $d_{E}$ which is clearly quasi-isometric to $d_{H}$. $d_{E}$ is defined by adding a new vertex $h$ to $C(\Sigma)$ for each handlebody $H$, $\partial H=\Sigma$, and adjoining a length $=1$ edges between each scc in $D(H)$ and $h$. They prove that for genus $\Sigma>2, \operatorname{diam}_{d_{E}}=\infty$. Thus we have:

Theorem 3.3. $\operatorname{diam}_{d_{H}}(C(\Sigma))=\infty$, for genus $(\Sigma) \geq 2$.

Since $\operatorname{diam}_{d_{H}}(C(\Sigma))=\infty$, Theorems 3.1 and 3.2 obstruct certain -in some sense, most-Morse functions, or Heegaard decompositions $M=X \cup_{\Sigma} Y$ from arising via embedding $M \hookrightarrow N \times \mathbb{R}$ for $N=S^{3}$, or more generally $N$ closed, reducible, and containing no incompressible surface.

\section{Acknowledgements}

The first author acknowledges support by NSF grant DMS-1105738.

The second author would like to acknowledge stimulating discussion with Cameron Gordon on this subject.

\section{References}

[1] Ryan Budney and Benjamin Burton, Embeddings of 3-manifolds in $S^{4}$ from the point of view of the 11-tetrahedron census, (2008), arXiv:0810.2346.

[2] Andrew Casson and Cameron Gordon, Reducing Heegaard splittings, Topology and its Applications 27(3)(1987), 275-283.

[3] Ralph H. Fox, On the imbedding of polyhedra in 3-space, Annals of Mathematics 49(1948), 462-470.

[4] W. J. Harvey, Boundary structure of the modular group, In Riemann surfaces and related topics: Proceedings of the 1978 Stony Brook Conference (State Univ. New York, Stony Brook, N.Y., 1978), Ann. of Math. Stud. 97, Princeton Univ. Press, Princeton, N.J., (1981), 245-251.

[5] John Hempel, 3-manifolds as viewed from the curve complex, Topology 40(3)(2001), 631-657.

[6] Joseph Maher and Saul Schleimer, The compression body graph has infinite diameter, Algebr. Geom. Topol. 21(4)(2021), 1817-1856. doi 10.2140/agt.2021.21.1817

[7] Friedhelm Waldhausen, Heegaard-Zerlegungen der 3-sphäre, Topology $\mathbf{7}(\mathbf{2})(1968), 195-203$. 


\author{
Ian Agol \\ Department of Mathematics \\ University of California, Berkeley \\ Berkeley, CA 94720 \\ ianagol@math.berkeley.edu \\ Michael Freedman \\ Microsoft Research, Station Q, and Department of Mathematics \\ University of California, Santa Barbara \\ Santa Barbara, CA 93106 \\ mfreedman@math.ucsb.edu
}

